\title{
Antibody recognition of histone post-translational modifications: emerging issues and future prospects
}

\begin{abstract}
"...it is fair to say that much of the advancement in our understanding of chromatin modifications and chromatin structure would not have been possible without the development of post-translational modification-specific antibodies."
\end{abstract}

\section{KEYWORDS: antibody $=$ antibody specificity $\approx$ chromatin $\approx$ histones - post-translational modifications}

Last year marked the 10-year anniversary of the 'histone code' hypothesis, which formally proposed that combinatorial post-translational modifications (PTMs) on histone proteins govern the diverse activities associated with eukaryotic DNA [1,2]. In the intervening decade, significant advancements have been made in our understanding of chromatin biology. For example, more than 60 sites of modification on histones have been identified (which include acetylation, phosphorylation, ubiquitination, methylation and others), as have a variety of specialized effector domains that recognize these histone PTMs [3,4]. The collective results of many studies show that histone PTMs contribute to a wide range of biological processes, including gene transcription, DNA replication and DNA repair. These advances, however, would not have been possible without the creation of PTM-specific histone antibodies $[5,6]$. Histone antibodies have become the predominant tool for chromatin research, and are ubiquitously used to understand how histone PTMs regulate chromatin structure and function.

While histone antibodies are integral tools for chromatin research, several issues regarding their future utility have emerged. More than 1000 different antibodies raised against histone PTMs are commercially available. The process of choosing the right antibody for a particular application can be both daunting and expensive. Often, minimal information is available regarding the substrate against which these reagents were developed, and the long-term reliability of polyclonal antibodies is most often limited by the nonrenewability of particularly effective lots. To further complicate matters, recent findings have highlighted the influence of combinatorial PTMs on the recognition and binding of these antibodies [7-9]. How then do we begin to assess antibody specificity, and will future technologies be developed to aid in the detection and study of histone PTMs? Below, we summarize some of the recent lessons learned and future technological advances that will likely improve antibody-mediated histone PTM studies.

Researchers have long known that histone antibodies are not always specific for their intended PTM targets, and often recognize 'off-target' proteins and PTMs. Until recently, the primary approaches used to characterize the specificity of histone antibodies were ELISAs, western blot analyses, chromatin immunoprecipitation (ChIP) and immunofluorescence-based assays. Typically, PTM-specific antibodies are first screened in ELISAs with the PTM-modified immunogenic peptide and further validated by peptide competition. In addition, antibodies are sometimes examined for their ability to detect off-target PTMs using peptides that harbor PTMs at different residues that might be potential targets of the antibody (e.g., the ability of a H4K5 acetylation-specific antibody to detect H4K8 acetylation and so forth). Ideally, these antibodies are also validated in a genetically amenable model organism (such as yeast) by examining a mutated target protein that harbors a precise amino acid substitution at the modified residue (e.g., H4K5 $\rightarrow$ H4A5). To date, however, there are no uniform approaches for antibody characterization. Furthermore, characterizations performed on many commercial histone antibodies are limited to their ability to detect a target PTM in a single organism under select assay conditions. While these methods have generally been sufficient (when employed properly), they still do not report on more complex, but relevant issues, such as antibody specificity in the context of adjacent PTMs.

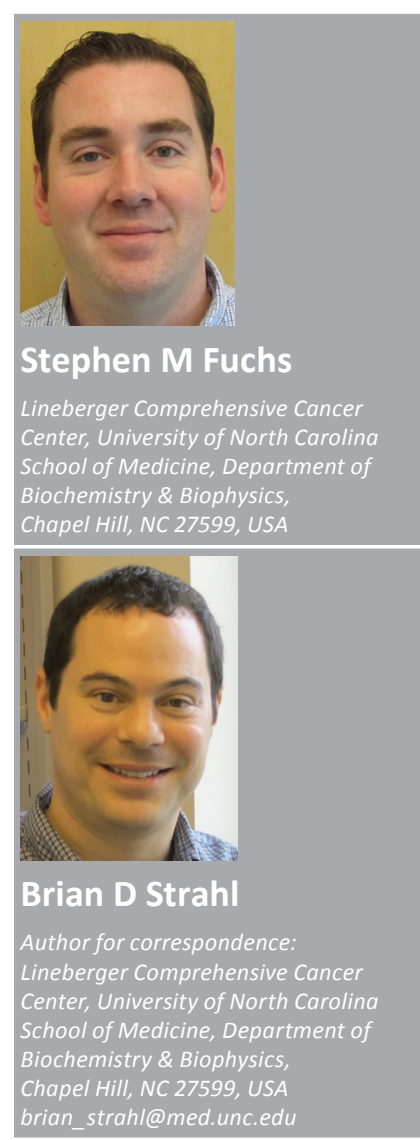

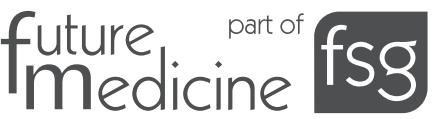


Studies using peptide array platforms, along with a large-scale initiative by the modENCODE consortium, have revealed some alarming results about histone antibody specificity [7-9]. Lieb and colleagues of the modENCODE consortium performed a large and systematic characterization of over 200 widely used commercial antibodies and found that a high percentage of these antibodies were incompatible with at least one standard technique (including western blot analysis and ChIP-seq). This information has been made available as an online resource for finding suitable antibodies $[8,101]$. In addition, studies using combinatorially modified peptide library arrays have found that: antibodies intended to recognize methyllysine often have difficulty correctly distinguishing the correct methyl state (mono-, di- or tri-methyl), many antibodies recognize the correct PTM but without the desired sequence context, and many antibodies are influenced by PTMs adjacent to their particular epitope $[7,9]$. Recognizing the wrong sequence is an obvious weakness of any antibody, but not being able to recognize the difference between two and three methyl groups on a lysine side chain may result in the inability to properly distinguish between the recruitment of a transcriptional repressor complex instead of a transcriptional activator complex $[10,11]$. The prevalence and importance of neighboring PTMs is only beginning to be understood, and there are now several clear examples of neighboring phosphorylation marks negatively affecting the recognition of methyllysine binding proteins such as HP1, TAF3 or CHD1 $[9,12,13]$.

The numerous examples of antibody promiscuity discovered to date clearly underscore the need for more rigorous antibody validation. One question to ask then is who should be responsible for increased testing - the user or the companies marketing these antibodies? On one hand, with technologies now available to rapidly screen antibodies against a wide array of peptide targets, there should be no reason a commercial antibody should not be analyzed in this detail and if needed, affinity purified until it exhibits specificity for its intended target. However, further testing to include all combinations of neighboring PTMs would undoubtedly increase the costs of antibodies - especially when you consider that each lot would need to be tested. Furthermore, it will be nearly impossible for companies to predict all the applications in which their products would be used and thus, at some point, validation for specialized applications should fall upon the user.

Regardless of the concerns highlighted above, histone antibodies will remain an important and essential tool for chromatin research. However, as biological questions regarding the histone code (i.e., combinatorial PTMs) become more complex, it is a fair question to ask whether antibodies will continue to be the primary tools of the chromatin biologist, or if the field will look to more specialized reagents and techniques. New solutions for histone PTM detection may lie in alternative strategies for macromolecular recognition. Recently, DNA aptamer technology was used to isolate an oligonucleotide capable of recognizing acetylation at K16 of histone H4 [14]. Aptamers are extremely appealing, as they are inexpensive and completely renewable. Similarly, several small molecules have been synthesized which selectively recognized lysine methylation $[15,16]$. Small molecules may have promise for recognizing PTMs in a sequence-independent manner, which would have great utility as affinity purification or enrichment reagents, and appropriate small molecules may permeate living cells, expanding potential utility. Furthermore, as recombinant and synthetic antibodies as well as antibody mimetics are becoming more widely used [17], the possibility of producing PTM-specific reagents is perhaps more feasible. These approaches offer noticeable and significant advantages over traditional antibodies in that they are largely synthetic and reproducible, not relying on animals for production. However, the ability to achieve either selectivity or specificity nearing that of antibodies has yet to be routinely achieved.

\section{"The numerous examples of antibody promiscuity discovered to date clearly underscore the need for more rigorous antibody validation."}

Another growing approach to combinatorial detection of histone PTMs is in the use of mass spectrometry (MS). Today, MS is the primary tool for identifying new PTMs on histones. Using both top-down and bottomup approaches, researchers are not only able to identify low-abundance PTMs but also identify combinations of modifications that occur on the same protein [18]. Recently, MS analysis has been used to identify specific PTM patterns associated with a replication origin in yeast, suggesting that 
this technology may be used to characterize precise patterns of PTMs at individual chromatin loci or domains [19]. However, MS analysis is still a specialized field which is both time consuming and costly. As such, it will need to be paired with other probes such as antibodies for more routine biochemical studies such as western blotting.

"...today we are nearing a potential impasse, where our knowledge of histone PTMs is great enough that the tools that brought us here are no longer sufficient."

While concerns about histone antibody specificity (and nonhistone antibodies) are not new, it is fair to say that much of the advancement in our understanding of chromatin modifications and chromatin structure would not have been possible without the development of PTM-specific antibodies. Yet today we are nearing a potential impasse, where our knowledge of histone PTMs is great enough that the tools that brought us here are no longer sufficient. Our ability to find new and creative solutions to these structure-function problems will dictate the rate of progress on our journey to understand all aspects of how the cell regulates access to our DNA.

\section{Acknowledgements}

The authors thank Stephen Frye, Marcey Waters, DeepakJha, Scott Rothbart and Glenn Wozniak for helpful discussions and critical reading of the manuscript.

\section{Financial \& competing interests disclosure}

Work in the Strabl laboratory is funded by grants from the National Institutes of Health. The authors have no other relevant affiliations or financial involvement with any organization or entity with a financial interest in or financial conflict with the subject matter or materials discussed in the manuscript apart from those disclosed.

No writing assistance was utilized in the production of this manuscript.

\section{Bibliography}

1 Gardner KE, Allis CD, Strahl BD: OPERating ON chromatin, a colorful language where context matters. J. Mol. Biol. 409(1), 36-46 (2011).

2 Strahl BD, Allis CD: The language of covalent histone modifications. Nature 403(6765), 41-45 (2000).

3 Kouzarides T: Chromatin modifications and their function. Cell 128(4), 693-705 (2007).

4 Ruthenburg AJ, Allis CD, Wysocka J: Methylation of lysine 4 on histone H3: intricacy of writing and reading a single epigenetic mark. Mol. Cell 25(1), 15-30 (2007).

5 Perez-burgos L, Peters AH, Opravil S et al: Generation and characterization of methyllysine histone antibodies. Methods Enzymol. 376, 234-254 (2004).

6 Sarma K, Nishioka K, Reinberg D: Tips in analyzing antibodies directed against specific histone tail modifications. Methods Enzymol. 376, 255-269 (2004).

7 Bock I, Dhayalan A, Kudithipudi S et al.: Detailed specificity analysis of antibodies binding to modified histone tails with peptide arrays. Epigenetics 6(2), 256-263 (2011).

8 Egelhofer TA, Minoda A, Klugman S et al.: An assessment of histone-modification antibody quality. Nat. Struct. Mol. Biol. 18(1), 91-93 (2011).
9 Fuchs SM, Krajewski K, Baker RW et al: Influence of combinatorial histone modifications on antibody and effector protein recognition. Curr. Biol. 21(1), 53-58 (2011).

10 Kim T, Buratowski S: Dimethylation of H3K 4 by Set 1 recruits the Set 3 histone deacetylase complex to $5^{\prime}$ transcribed regions. Cell 137(2), 259-272 (2009).

11 Taverna SD, Ilin S, Rogers RS et al.: Yng1 $\mathrm{PHD}$ finger binding to $\mathrm{H} 3$ trimethylated at K4 promotes NuA3 HAT activity at K14 of $\mathrm{H} 3$ and transcription at a subset of targeted ORFs. Mol. Cell 24(5), 785-796 (2006).

12 Fischle W, Tseng BS, Dormann HL et al.: Regulation of HP1-chromatin binding by histone $\mathrm{H} 3$ methylation and phosphorylation. Nature 438(7071), 1116-1122 (2005).

13 Varier RA, Outchkourov NS, De Graaf P et al:: A phospho/methyl switch at histone $\mathrm{H} 3$ regulates TFIID association with mitotic chromosomes. EMBO J. 29(23), 3967-3978 (2010).

14 Williams BA, Lin L, Lindsay SM et al: Evolution of a histone H4-K16 acetyl-specific DNA aptamer. J. Am. Chem. Soc. 131(18), 6330-6331 (2009).

15 Beshara CS, Jones CE, Daze KD et al.: A simple calixarene recognizes post-translationally methylated lysine. Chembiochem 11(1), 63-66 (2010).
16 Ingerman LA, Cuellar ME, Waters ML: A small molecule receptor that selectively recognizes trimethyl lysine in a histone peptide with native protein-like affinity. Chem. Commun. (Camb.) 46(11), 1839-1841 (2010).

17 Fellouse FA, Esaki K, Birtalan S et al.: High-throughput generation of synthetic antibodies from highly functional minimalist phage-displayed libraries. J. Mol. Biol. 373(4), 924-940 (2007).

18 Young NL, Dimaggio PA, Plazasmayorca MD et al.: High throughput characterization of combinatorial histone codes. Mol. Cell. Proteomics 8(10), 2266-2284 (2009).

19 Unnikrishnan A, Gafken PR, Tsukiyama T: Dynamic changes in histone acetylation regulate origins of DNA replication. Nat. Struct. Mol. Biol. 17(4), 430-437 (2010).

\section{Website}

101 Antibody validation database http://compbio.med.harvard.edu/antibodies/ 